\title{
Proposed Mass Spectroscopy Method to Facilitate the Observation of Primordial Superheavy Nuclei
}

\author{
Joseph Bevelacqua
}

Funding: The author(s) received no specific funding for this work.

Potential competing interests: The author(s) declared that no potential competing interests exist.

\begin{abstract}
A proposed experimental method to facilitate the observation of postulated primordial superheavy nuclei is investigated using mass spectroscopy. Detection of primordial superheavy systems by detailed investigation of lead ions is motivated by the fact that the 232Th, $235 \mathrm{U}$, and $238 \mathrm{U}$ natural decay series and transuranic elements have decay pathways that terminate in the $Z=82$ system, and the presumption that the postulated superheavy systems will also decay into lead isotopes. Although extreme sensitivity would be required, primordial superheavy systems would present a unique signature in a mass spectroscopy experiment utilizing lead ions.
\end{abstract}

\subsection{Introduction}

The investigation of the stability of superheavy nuclei has been a continuing area of active experimental and theoretical interest ${ }^{1-30}$. These studies extend well beyond the current Periodic Table limit of the $\mathrm{Z}=118$ Oganesson system. In particular, the author has investigated the possibility of much heavier systems using a single-particle model and finds several possible islands of stability in the $A=400-1300$ mass region $5,21-30$.

The stability of superheavy systems are evaluated by calculating single-particle neutron and proton levels using a methodology previously used to investigate $A=298-472$ doubly-closed shell nuclei ${ }^{5}$ and nuclear systems in the $570 \leq A$ $\leq 620^{21}, 620<\mathrm{A}<700^{22}, 700 \leq \mathrm{A}<800^{23}, 800 \leq \mathrm{A}<900^{25}, 900 \leq \mathrm{A}<1000^{26}, 1000 \leq \mathrm{A}<1100^{27}, 1100 \leq \mathrm{A}<1200^{28}$, and $1200 \leq \mathrm{A}<1300^{30}$ mass regions.

The method for calculating single-particle energies is well established ${ }^{3,5,21-30}$. Details of the methodology were provided in Ref. 21 , which extended the approach of Petrovich et al. ${ }^{5}$ Specific details of the numerical method, model, and convergence criteria are provided in Refs. 2, 5, 21-33.

The model potential varies with the nucleus mass and is derived from Rost interaction ${ }^{2,24,29}$. It is extrapolated from $Z \leq 82$ data without the benefit of experimental benchmarks in the superheavy mass region. Although this is a necessity due to the lack of experimental data, it must be acknowledged as a weakness in the present approach. This weakness will be applicable for any current theoretical investigation in the superheavy mass region. Nuclear stability with respect to alpha decay, beta decay, positron decay, and electron capture is addressed using the method previously published by the author and coworkers ${ }^{5,21-29,30}$ that is similar to the approach of Ref. 3 . Spontaneous fission stability is expected to be enhanced near doubly-closed shells. Detailed calculations of the spontaneous fission half-lives of $400 \leq \mathrm{A}$ 
$<1300$ nuclei have not been attempted ${ }^{5,21-30}$. However, estimates using the Wentzel-Kramers-Brillouin (WKB) approximation methodology and the phenomenological parameter values of Ref. 3 suggest fission half-lives near closed shells are much greater than the effective decay half-lives. This conclusion is consistent with the doubly-closed shell $\mathrm{A}=$ 298 - 472 nuclei values of Refs. 3 and 5.

As used in this paper, an effective half-life $(T)$ includes the combined effect of the alpha and beta decay modes that dominate decays in the superheavy mass region ${ }^{5,21-30:}$

$$
T(\text { eff })=\frac{T(\alpha) T(\beta)}{T(\alpha)+T(\beta)}
$$

Most of the $400 \leq A<1300$ systems have effective half-lives less than a second ${ }^{5,21-30}$. The calculated half-lives of most the investigated nuclei are shorter than the observed half-lives in $Z=114-118$ systems $^{34}$.

The single-particle model and associated extrapolated potential ${ }^{24,29}$ lead to interesting results that suggest the possibility of islands of stability in the $400 \leq A<1200$ mass region. These stability islands are characterized by the notation ( $\left.Z, A, T_{1 / 2}\right)$ where $Z$ is the atomic number, $A$ the mass number, and $T_{1 / 2}$ the half-life. Most of these islands have effective half-lives that are in the $s-y$ range with $(274,888,590 y)^{25}$ having the longest half-life value in the $400 \leq A<$ 1200 mass region. However, the $1200 \leq \mathrm{A}<1300$ superheavy nuclei ${ }^{30}$ exhibit stability islands that contain long-lived or primordial superheavy nuclei (PSN) with half-lives that are comparable with the age of the universe. In particular, the following nuclear systems ${ }^{30}$ motivate this paper: $\left(354,1216,2.7 \times 10^{10} \mathrm{y}\right),\left(354,1218,8.0 \times 10^{10} \mathrm{y}\right),\left(354,1220,2.5 \times 10^{11} \mathrm{y}\right)$, $\left(354,1222,5.1 \times 10^{11} y\right),\left(354,1224,1.5 \times 10^{12} y\right),\left(354,1226,4.8 \times 10^{12} y\right),\left(360,1262,1.2 \times 10^{10} y\right)$, and $\left(368,1290,2.5 \times 10^{10}\right.$ y). Each of these systems is beta stable and decays by alpha emission. These primordial superheavy systems and their possible experimental observation are the subject of this paper.

\subsection{Basis for Experimental Investigation}

The reader is cautioned that the proposed PSN are based on calculations using a potential model $\mathrm{l}^{2,24,29}$ that is extended well beyond its experimental basis. Previous papers ${ }^{5,21-30}$ cautioned readers that the calculated half-lives are quite uncertain, but the locations of possible islands of stability are likely to be more reliable. With these caveats, singleparticle model calculations suggest the possible existence of $\mathrm{PSN}^{30}$, and this paper investigates possible experimental methods to facilitate their observation.

\subsection{Model Weaknesses}

In Ref. 30, eight $1200 \leq \mathrm{A}<1300$ PSN with half-lives $>10^{10}$ y were suggested by the model. These half-lives are on the order of or greater than the current estimate for the age of the Universe $\left(\approx 1.4 \times 10^{10} \mathrm{y}\right)$, which is unexpected for a superheavy system. If these long-lived nuclei were ever created, and had no other more rapid decay processes, they would presumably still exist on earth today. The proposed model does not account for the possibility that as the nucleus numbers $\mathrm{A}, \mathrm{N}$, and $\mathrm{Z}$ become larger, new, more rapid decay modes could exist. These decay modes would then be more likely to dominate all decay processes of these superheavy systems. This is a significant weakness of the extension of the theory beyond its origin via connection to known isotopes $5,21-30$.

Another weakness of the theoretical approach used as the basis for this paper is treating all evaluated nuclei as spherically symmetric systems. Many of these systems are likely deformed and these deformations should be included in subsequent investigations. These calculations have been initiated. However, it seems unlikely that any given $A(N, Z)$ 
nuclear system with a deformed structure will be more stable than the spherically symmetric configuration utilized in the model. Accordingly, deformed systems would likely have half-lived that are shorter than the corresponding spherical systems noted in Section 1.0.

These limitations and uncertainties in the model interaction preclude the absolute determinations of single-particle energies, $Q$ values, and half-lives. The model does facilitate a comparison of the relative stability of nuclear systems and identification of possible islands of stability. These limitations are unavoidable given the lack of experimental data and uncertainties in the model and supporting nuclear interaction ${ }^{29}$. The adjusted Rost interaction formulated in Ref. 29 accounted for the uncertainties in the potential strength noted in studies of a wide range of nuclear systems.

The aforementioned weaknesses are difficult to assess, but the model prediction for the most stable $1200 \leq \mathrm{A}<$ 1300 PSN $\left(354,1226,4.8 \times 10^{12} y\right)$ can be partially assessed by comparing the (A, Z) values of this system to the predictions of Adler's relationship 35,36 that provides the most stable nucleus $Z$ value for a given $A$ :

$Z={ }^{\frac{0.487 A}{A^{\frac{A^{2 / 3}}{166}}}}$

This relationship suggests that the $A=1226$ system should be most stable for a $Z$ value of 353 which is in reasonable agreement of the $Z=354$ result obtained by the model for the $\left(354,1226,4.8 \times 10^{12} y\right) P S N$. Although qualitative, the reasonable comparison between the model and predictions of the Adler relationship of Eq. 2 serves to place a portion of the model weakness issues into perspective.

\subsection{Experimental Verification}

$Z=114$ to 118 superheavy nuclei have been created through fusion reactions between ${ }^{48} \mathrm{Ca}$ beams and actinide $\operatorname{targets}^{19}$. Creation of elements with $Z>118$ likely requires projectiles with $Z>20$. These investigations have yet to be successful. The creation of $400 \leq A<1300$ superheavy systems is significantly more complex than the near term challenge of synthesizing $Z>118$ nuclei. Possible experimental techniques to observe these superheavy systems include: (1) multiple beam collisions, (2) detection of high alpha particle decay energies, (3) laser resonance chromatography, and (4) observation of trace primordial superheavy elements in lead using mass spectroscopy. The discussion in Sections 4.1 -4.4 is based on the presumption that the PSN addressed in Ref. 30 exist.

\subsection{Multiple Beam Collisions}

Conventional binary collision processes involving heavy ions beams are not currently capable of reaching the $400 \leq$ $\mathrm{A}<1300$ mass region. For example, ${ }^{285} \mathrm{Cn}$ has a half-life of about $34 \mathrm{~s}^{34}$. Even if it were possible to perform a ${ }^{285} \mathrm{Cn}$ $+{ }^{285} \mathrm{Cn}$ collision, it would not produce a PSN system. Experimental investigation of $1200 \leq \mathrm{A}<1300$ primordial superheavy systems requires a novel approach. For example, simultaneously colliding six ${ }^{238} \mathrm{U}$ ions theoretically reaches the $1200 \leq A<1300$ mass region, but this approach is not yet viable. Experimental techniques would need to be developed to facilitate the collision and fusion of multiple ${ }^{238} \mathrm{U}$ beams to create $1200 \leq A<1300$ systems. Although this may be eventually possible, it is not a likely approach to successfully observe primordial superheavy systems in the near term.

\subsection{Detection of High Alpha Particle Decay Energies}

A second possible experimental approach is offered by the high alpha particle energies emitted by the postulated $1200 \leq \mathrm{A}<1300$ systems $^{30}$. The alpha particle energies of these theoretical superheavy nuclei are more than $100 \%$ larger 
than the measured $Z=114-118$ values $^{34}$. This substantial increase in alpha particle energies offers a possible avenue for the experimental observation of $1200 \leq A<1300$ nuclei.

Compared to $Z=114$ - 118 nuclei, the higher alpha particle energies from the $1200 \leq A<1300$ systems have a longer range in a material medium. This range manifests itself as a longer track length as the alpha particle is attenuated by the medium. Measuring alpha track lengths is a well-established approach in applied physics including the measurement of the ${ }^{222} \mathrm{Rn}$ air concentration ${ }^{36,37}$. Since the track length is related to the alpha particle energy, it provides a possible method to investigate the existence of the $1200 \leq A<1300$ primordial superheavy systems ${ }^{30}$.

Although the alpha track method is technically feasible, it would require a sample of superheavy material. Obtaining this sample depends on the existence of the aforementioned superheavy systems in sufficient quantity to permit observation via energy deposition that produces the track. Since obtaining a sufficiently large sample to produce observable tracks is unlikely, this approach is not a likely near term solution to the observation of $1200 \leq A<1300$ primordial superheavy systems.

\subsection{Laser Resonance Chromatography}

Optical spectroscopy provides an established technique to obtain basic structure data for an atomic system. Both fluorescence and resonance ion spectroscopy enable the identification of optical spectra wavelengths for elements up to nobelium $(Z=102)^{38}$. As the $Z$ value increases, the refractory nature of these systems decreases the production yields. This decrease in yield challenges the current optical detection limits.

To overcome this sensitivity limitation, a new approach of ion-mobility assisted laser spectroscopy has been proposed $^{38}$. This approach offers the capability of broad-band level searches and high-resolution hyperfine spectroscopy, and has the potential to extend the laser resonance chromatography technique beyond $Z=102$. Since this technique has not yet been demonstrated for $Z>102$ systems, it is unlikely to provide a near-term technique for the detection of the 1200 $\leq \mathrm{A}<1300$ PSN systems noted in Ref. 30 .

\subsection{Observation of Primordial Superheavy Elements in Lead}

A final possible experimental verification approach is based on the fact that various lead isotopes are the end state of all natural decay chains ${ }^{36,37}$. These decay chains include the ${ }^{232} \mathrm{Th},{ }^{235} \mathrm{U}$, and ${ }^{238} \mathrm{U}$ natural decay series that terminate in ${ }^{208} \mathrm{~Pb},{ }^{207} \mathrm{~Pb}$, and ${ }^{206} \mathrm{~Pb}$, respectively ${ }^{36,37}$. Transuranic systems also decay into lead isotopes through a variety of decay modes ${ }^{39,40}$. For example, the following transuranic systems decay into one of the natural decay series that eventually terminate in a stable lead isotope ${ }^{39,40:}{ }^{250} \mathrm{Cf} \rightarrow{ }^{246} \mathrm{Cm} \rightarrow{ }^{242} \mathrm{Pu} \rightarrow{ }^{238} \mathrm{U}$ and ${ }^{252} \mathrm{Cf} \rightarrow{ }^{248} \mathrm{Cm} \rightarrow{ }^{244} \mathrm{Pu} \rightarrow{ }^{240} \mathrm{U} \rightarrow$ ${ }^{240} \mathrm{~Np} \rightarrow{ }^{240} \mathrm{Pu} \rightarrow{ }^{236} \mathrm{U} \rightarrow{ }^{232} \mathrm{Th}$. Heavier systems (e.g., ${ }^{293} \mathrm{Og}$ ) also decay into stable lead isotopes through a series of alpha, beta, and cluster decay modes. For example, ${ }^{293} \mathrm{Og}$ decays through a number of decay pathways including a decay mode to ${ }^{207} \mathrm{~Pb}:{ }^{293} \mathrm{Og} \rightarrow{ }^{289} \mathrm{Lv} \rightarrow{ }^{285} \mathrm{Fl} \rightarrow{ }^{281} \mathrm{Cn} \rightarrow{ }^{277} \mathrm{Ds} \rightarrow{ }^{273} \mathrm{Hs} \rightarrow{ }^{269} \mathrm{Sg} \rightarrow{ }^{265} \mathrm{Rf} \rightarrow{ }^{261} \mathrm{No} \rightarrow{ }^{257} \mathrm{Fm} \rightarrow{ }^{253} \mathrm{Cf} \rightarrow{ }^{249} \mathrm{Cm} \rightarrow$ ${ }^{249} \mathrm{Bk} \rightarrow{ }^{245} \mathrm{Am} \rightarrow{ }^{245} \mathrm{Cm} \rightarrow{ }^{241} \mathrm{Pu} \rightarrow{ }^{237} \mathrm{U} \rightarrow{ }^{237} \mathrm{~Np} \rightarrow{ }^{233} \mathrm{~Pa} \rightarrow{ }^{233} \mathrm{U} \rightarrow{ }^{229} \mathrm{Th} \rightarrow{ }^{225} \mathrm{Ra} \rightarrow{ }^{225} \mathrm{Ac} \rightarrow{ }^{221} \mathrm{Fr} \rightarrow{ }^{221} \mathrm{Ra} \rightarrow{ }^{207} \mathrm{~Pb}$. The last ${ }^{293} \mathrm{Og}$ decay step involves the emission of a ${ }^{14} \mathrm{C}$ cluster. Additional examples of these more complex decay sequences and the lead isotope produced include ${ }^{292} \mathrm{Ts}\left({ }^{206} \mathrm{~Pb}\right.$ and $\left.{ }^{208} \mathrm{~Pb}\right),{ }^{273} \mathrm{Ds}\left({ }^{207} \mathrm{~Pb}\right)$, and ${ }^{288} \mathrm{Mc}\left({ }^{206} \mathrm{~Pb}\right.$ and $\left.{ }^{208} \mathrm{~Pb}\right)$. These heavy nucleus decay chains are complex, involve multiple pathways, and can produce multiple lead isotopes as a result of the alpha, beta, and cluster decay modes 39,40 . Therefore, it is reasonable to postulate that superheavy systems will also decay into a stable isotope of lead. If the PSN decays terminate in a stable element of lead as the end product of 
its decay through alpha, beta, and cluster modes, then these decay pathways form the basis for the possibility of finding trace quantities of primordial superheavy systems in lead.

Using analogous arguments, the PSN summarized in Section $1.0^{30}$, can also be postulated to decay into stable lead isotopes. Given the fact that the postulated $\mathrm{PSN}^{30}$ are even-even nuclei, it is possible that they will decay into ${ }^{206} \mathrm{~Pb}$ and $/$ or ${ }^{208} \mathrm{~Pb}$ depending on the particular decay mode. If these PSN do indeed exist and if the decays terminate in a stable lead isotope, it is theoretically possible to experimentally detect them using mass spectroscopy.

The reader should note that the proposed experimental investigation is based on the following assumptions:

1. Primordial superheavy elements with lifetimes on the order of the age of the universe exist. This assumption is based on single-particle model calculations in the superheavy mass region ${ }^{5,21-30}$.

2. PSNs decay by the alpha, beta, and cluster decay modes. This assumption is supported by the behavior of the ${ }^{232} \mathrm{Th}$, ${ }^{235} \mathrm{U}$, and ${ }^{238} \mathrm{U}$ natural decay series ${ }^{36,37}$, the decay of transuranic nuclides ${ }^{39,40}$, and the decay modes noted in singleparticle model calculations in the superheavy mass region ${ }^{5,21-30}$.

3. No unique, rapid PSN decay modes occur that would decrease the half-lives of the systems modeled in Refs. 5, 21-30.

4. The PSN decay chains terminate in a stable isotope of lead.

5. Lead samples consist of stable $Z=82$ isotopes and remnant PSNs.

If lead targets were vaporized, and then accelerated in a charged particle accelerator, any remnant primordial superheavy elements could be separated from the dominant lead isotopes on the basis of mass. Within this mass spectrum, there could be the remnants of the long-lived parent superheavy nuclei. Extreme precision would be required to detect these primordial superheavy trace isotopes. At the very least, an experimental bound could be placed on the existence of the superheavy isotopes noted in Ref 30. This is an interesting possibility for an experimental technique, but sufficient sensitivity would be required. The requirements for extreme sensitivity in the observation of PSNs are similar to the challenges involved with ongoing neutrino oscillation experiments that investigate $\mathrm{CP}$ violation.

The use of mass spectroscopy to investigate the existence of PSN is discussed in the next section. This approach presents a well-established technique, but will be a significant experimental challenge. However, it is a near-term possibility that merits further investigation.

\subsection{Mass Spectroscopy}

A mass spectrometer is a device that is designed to sort the mass of particles in an ion beam. In a mass spectrometer, charged particles generated by an ion source are accelerated through a potential difference. The ions are subjected to a uniform magnetic field $(B)$ that is orthogonal to the direction of their motion. Through the action of the magnetic force, the ion's trajectory assumes a circular arc and strikes a photographic plate or exits through an aperture to a detector. The radius of the arc is proportional to the square root of the mass of the ion (Eq. 6).

Classical physics is sufficient to define the characteristics of a basic mass spectrometer. Assuming an ion with charge $\mathrm{q}$ and mass $\mathrm{m}$ starts from rest and is accelerated through a potential difference $\Delta \mathrm{V}$, the resulting kinetic energy $\mathrm{T}$ is given by

$$
T={ }^{\frac{1}{2}} m v^{2}
$$


which is equivalent to their loss of potential energy

1

${ }^{\frac{1}{2}} m v^{2}=q \Delta V$

The ions move in a circular radius $r$ with velocity $v$ determined by equating the magnetic force and centrifugal force $\frac{m v^{2}}{r}=q v B$

The velocity v from Eq. 4 is inserted into Eq. 5 to determine the radius of the ion's arc:

$r=\left[\frac{2 m \Delta V}{q B^{2}}\right]_{1 / 2}$

For identical values of $\mathrm{q}, \Delta \mathrm{V}$, and $\mathrm{B}$, the ratio of radii for two ions of different masses is:

$\frac{r_{2}}{r_{1}}=\left[\frac{m_{2}}{m_{1}}\right]^{1 / 2}$

Consider a reference mass spectrometer that utilizes an electric field strength of $3000 \mathrm{~V}$ and a magnetic field strength of $0.12 \mathrm{~T}$. For specificity, assume that both the lead isotopes and the PSN are singly ionized with a charge of +1 e, and the lead beam contains the most stable PSN of Ref. 30 (i.e., $\left(354,1226,4.8 \times 10^{12} \mathrm{y}\right)$ ). In this spectrometer, the (354, $1226,4.8 \times 10^{12} \mathrm{y}$ ) nuclei and ${ }^{206} \mathrm{~Pb}$ and ${ }^{208} \mathrm{~Pb}$ lead isotopes would be detected at radii of about $2.3 \mathrm{~m}$ and $0.95 \mathrm{~m}$, respectively. The key question regarding the mass spectroscopy approach is the detection of the weak $Z=354$ signal (if it exists). However, the relative abundance of the superheavy element will be miniscule relative to the predominant lead signal. Although this would require extreme sensitivity to detect these primordial elements, the mass difference would be readily detected.

\subsection{Conclusions}

Calculations suggest the existence of PSNs in the vicinity of $Z=354^{30}$. Their postulated decay to a stable isotope of lead offers a possible approach for their detection. Mass spectroscopy techniques easily resolve the mass difference between primordial superheavy systems and stable isotopes of lead, if there is sufficient PSN present in the lead sample used to produce the ion beam. The success of the mass spectroscopy approach depends on the existence of the PSN and a spectrometer with sufficient sensitivity to detect the remnant superheavy nucleus signal.

\section{Acknowledgment}

The author would like to thank the unknown reviewer of Ref. 30 who suggested the possibility that primordial superheavy nuclei could decay into isotopes of lead. This reviewer comment motivated this paper and the possible development of a detection approach using a mass spectrometer.

\section{References}

1) C. Y. Wong, Phys. Lett. 21, 688 (1966).

2) E. Rost, Phys. Lett. 26B, 184 (1968).

3) A. Lukasiak and A. Sobiczewski, Acta Phys. Pol. B6, 147 (1975).

4) R. V. Gentry, T. A. Cahill, N. R. Fletcher, H. C. Kaufmann, L. R. Medsker, J. W. Nelson, and R. G. Flocchini, Phys. Rev.

Lett. 37, 11 (1976). 
5) F. Petrovich, R. J. Philpott, D. Robson, J. J. Bevelacqua, M. Golin, and D. Stanley, Phys. Rev. Lett. 37 , 558 (1976).

6) G. N. Flerov and G. M. Ter-Akopian, Rep. Prog. Phys. 46, 817 (1983).

7) R. Smolańczuk, Phys. Rev. C 56, 812 (1997).

8) M. Bender, K. Rutz, P.-G. Reinhard, J. A. Maruhn, and W. Greiner, Phys. Rev. C 58, 2126 (1998).

9) S. Hofmann and G. Münzenberg, Rev. Mod. Phys. 72, 733 (2000).

10) S. B. Duarte, O. A. P. Tavares, M. Gonçalves, O. Rodríguez, F. Guzmán, T. N. Barbosa, F. García, and A. Dimarco, J. Phys. G: Nucl. Part. Phys. 30, 1487 (2004).

11) H. Koura, T. Tachibana, M. Uno, and M. Yamada, Prog. Theor. Phys. 113, 305 (2005).

12) P. Mohr, Phys. Rev. C 73, 031301-1(R) (2006).

13) Yu. Ts. Oganessian et al., Phys. Rev. C 74, 044602 (2006).

14) P. R. Chowdhury, C. Samanta, and D. N. Basu, Phys. Rev. C 77, 044603 (2008).

15) C. Samanta, Prog. Part. Nucl. Phys. 62, 344 (2009).

16) P. Möller, A. J. Sierk, T. Ichikawa, A. Iwamoto, R. Bengtsson, H. Uhrenholt, and S. Åberg, Phys. Rev. C 79, 064304 (2009).

17) A. Marinov, Int. J. Mod. Phys. E 19, 131 (2010).

18) D. N. Poenaru, R. A. Gherghescu, and W. Greiner, Phys. Rev. Lett. 107, 062503 (2011).

19) Yu. Ts. Oganessian et al., Phys. Rev. Lett. 109, 162501 (2012).

20) K. Morita et al., J. Phys. Soc. Japan 81, 103201 (2012).

21) J. J. Bevelacqua, Physics Essays 25, 475 (2012).

22) J. J. Bevelacqua, Physics Essays 26, 516 (2013).

23) J. J. Bevelacqua, Physics Essays 27, 655 (2014).

24) J. J. Bevelacqua, Physics Essays 28, 300 (2015).

25) J. J. Bevelacqua, Physics Essays 29, 490 (2016).

26) J. J. Bevelacqua, Physics Essays 30, 1 (2017).

27) J. J. Bevelacqua, Physics Essays 30, 392 (2017).

28) J. J. Bevelacqua, Physics Essays 31, 235 (2018).

29) J. J. Bevelacqua, Physics Essays 31, 377 (2018).

30) J. J. Bevelacqua, Physics Essays 33, 276 (2020).

31) G. E. Brown, J. H. Gunn, and P. Gould, Nucl. Phys. 46, 598 (1963).

32) L. Fox and E. T. Godwin, Proc. Cambridge Philos. Soc. 45, 373 (1949).

33) J. Blomqvist and S. Wahlborn, Ark. Fys. 16, 545 (1959).

34) E. M. Baum, M. C. Ernesti, H. D. Knox, T. R. Miller, and A. M. Watson, Nuclides and Isotopes - Chart of the Nuclides, 17th ed (Knolls Atomic Power Laboratory, Schenectady, NY, 2010).

35) K. Adler, Coulomb Interactions with Heavy lons, CONF-720669, Proceedings of the Heavy lon Summer School, June 12 - July 1, 1972, Oak Ridge National Laboratory, Oak Ridge, TN (1972).

36) J. J. Bevelacqua, Contemporary Health Physics: Problems and Solutions, 2nd ed. (Wiley-VCH, Weinheim, 2009).

37) J. J. Bevelacqua, Basic Health Physics: Problems and Solutions, 2nd ed. (Wiley-VCH, Weinheim, 2010). 
38) M. Laatiaoui, A. A. Buchachenko, and L. A. Viehland, Phys. Rev. Lett. 125, 023002 (2020).

39) T. Gray, Isotope Decay Chains, https://periodictable.com/lsotopes.

40) Brookhaven National Laboratory, National Nuclear Data Center, https://www.nndc.bnl.gov/. 